Chinese Journal of Organic Chemistry

\title{
一种新的合成氯法拉滨的方法
}

\author{
夏 然 ${ }^{a, b}$ 郭 真 ${ }^{b}$ 秦博文 ${ }^{b}$ 汲智越 ${ }^{b}$ 谢明胜 ${ }^{b}$ \\ 渠桂荣*,, 郭海明*, $, a, b$ \\ ( ${ }^{a}$ 河南师范大学环境学院 新乡 453007) \\ ( ${ }^{b}$ 河南师范大学化学化工学院 新乡 453007)
}

\begin{abstract}
摘要 氯法拉滨是临床上唯一可以特异性地治疗儿童白血病的药物, 2004 年被美国食品和药物管理局批准上市. 由于 现有合成方法存在路线长、收率低和 $\alpha / \beta$ 异构体难以分离等问题，限制了该药物的广泛使用和进一步研究. 以廉价且商 品化的 2-氯腺苷为原料, 通过乙酸䣶保护糖环上的 3 个羟基, 在水合肼和乙酸作用下, 选择性地脱除 2 '位的乙酰基. 然 后，以商品化的氟代试剂二乙胺基三氟化硫(DAST)进行 2 '位羟基的氟代反应，再使用 $\mathrm{NH}_{3} / \mathrm{MeOH}$ 饱和溶液脱除乙酰基 可得氯法拉宾. 这 4 步反应的总收率为 $49 \%$, 且得到的产品全部为 $\beta$ 构型. 同时, 还发现 2 位取代基的位阻越大, 越有 利于选择性脱除 $2^{\prime}$ 位的乙酰基. 本方法可以在克级规模上进行生产, 收率无下降, 同时中间体及产物的纯化不需要通 过柱层析分离, 显示出良好的应用前景.
\end{abstract}

关键词 氯法拉滨; 2-氯腺苷; 氟代; 合成; 核苷

\section{A New Method for the Synthesis of Clofarabine}

\author{
Xia, Ran ${ }^{a, b} \quad$ Guo, Zhen ${ }^{b} \quad$ Qin, Bowen $^{b} \quad$ Ji, Zhiyue $^{b} \quad$ Xie, Mingsheng ${ }^{b}$ \\ Qu, Guirong*,a,b Guo, Haiming*,a,b \\ $\left({ }^{a}\right.$ School of Environment, Henan Normal University, Xinxiang 453007) \\ ( ${ }^{b}$ School of Chemistry and Chemical Engineering, Henan Normal University, Xinxiang 453007)
}

\begin{abstract}
Clofarabine is the active ingredient in the anti-pediatric leukemia drug, which was approved by U.S. Food and Drug Administration in 2004. However, the previous reported methods have long steps, low yield and difficult separation of $\alpha / \beta$ anomers, which restrict the wide use of the drug. In this manuscript, the cheap and commercial available 2-chloroadenosine was chose as the starting material to synthesize the clofarabine. By using acetic acid and hydrazine, the selective deprotection of acetyl group in 2'-position was accomplished. Subsequently, the fluorination step was realized by diethylaminosulfurtrifluoride (DAST). The clofarabine was synthesized with 4 steps in $49 \%$ total yield as a pure $\beta$-anomer. Meanwhile, the strong steric hindrance of 2-substitution was favorable for the 2'-deacetylation. Notably, the clofarobine could be synthesized at a gram scale using this method, which showed the good future of industrial application.
\end{abstract}

Keywords clofarabine; 2-chloroadenosine; fluorination; synthesis; nucleoside

糖基上带有氟原子的核苷类化合物具有优越的生 理和药理活性, 在临床上被广泛用作抗癌和抗病毒的药 物 ${ }^{[1,2]}$ (图 1). 因此, 该类化合物的合成受到化学工作者 广泛的关注. 其中, 氯法拉滨(Clofarabine, 2-chloro-2'deoxy-2'-arabinofluoroadenosine, 1) 是临床药物 Clolar 的 活性成分, 2004 年被美国食品和药物管理局(FDA)通过
快速评审通道批准上市, 用于治疗至少两种疗法无应 答，且没有其它疗法可选用的儿科急性淋巴细胞型白血 病. 它是目前唯一可以特异性地治疗儿童白血病的药 物. 此外, 该药物还可用于初次诊断为白血病的 21 岁及 以下患者的治疗. 该药物结合了白血病药物氟达拉滨和 克拉屈滨的优点，既抑制 DNA 聚合酶，又抑制核糖核

\footnotetext{
* E-mail: quguir@sina.com; guohm518@hotmail.com

Received January 14, 2014; revised February 22, 2014; published online March 3, 2014.

Project supported by the National Natural Science Foundation of China (Nos. 21072047, 21172059, 21272059, 21202039, 21372066), the Excellent Youth Foundation of Henan Scientific Committee (No. 114100510012), the Program for Innovative Research Team from the University of Henan Province (No. 2012IRTSTHN006), the Research Fund for the Doctoral Program of Higher Education (No. 20124104110006).

国家自然科学基金(Nos. 21072047, 21172059, 21272059, 21202039 和 21372066)、河南省杰出青年基金(No. 114100510012)、河南省高校科技创新研究 团队支持计划(No. 2012IRTSTHN006)、高等学校博士学科点专项科研基金(No. 20124104110006)资助项目.
} 


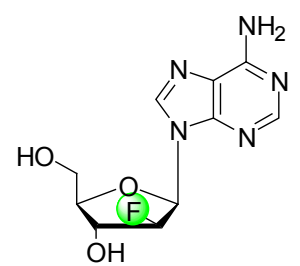
治疗白血病

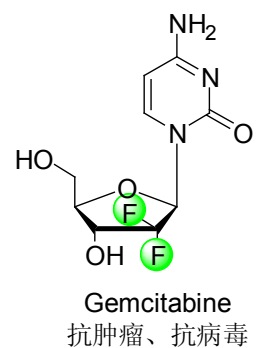

Clofarabine, 1

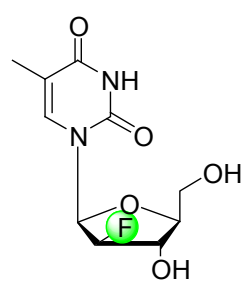

Clevudine 治疗乙型肝炎

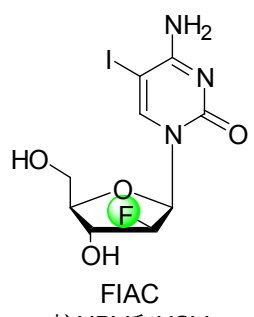

抗HBV和HSV
图 1 糖基上带有氟原子的核苷类药物

Figure 1 The selected nucleoside drugs with fluoride atom at the sugar ring

酸还原酶, 患者耐受性好, 无不可预知的不良反应 ${ }^{[3,4]}$.

目前，报道的氯法拉滨的合成方法主要包括以下 3 条路线(图 2). 1992 年, Montgomery 课题组 ${ }^{[5,6]}$ 首次实现 了氯法拉滨的全合成(图 2, 路线 a). 他们用 1-溴-2-脱
氧-2- $\beta$-氟-3- $O$-乙酰基-5- $O$-苯甲酰基- $D$-阿拉伯糖和 2,6二氯嘌呤缩合，再将 6 位氯原子氨解为氨基，随后脱除 糖环上的保护基, 最终分离 $\alpha, \beta$ 异构体, 得到氯法拉宾. 该路线一共 6 步反应，总收率仅为 6\%. 2004 年, ILEX 公司 (San Antonio, TX, USA) 对上述方法进行了改 进 ${ }^{[7,8]}$ (图 2, 路线 b). 他们采用单一 $\alpha$-构型的 1-溴-2-脱 氧- $2-\beta$-氟- 3,5 -二- $O$ - 苯甲酰基- $D$-阿拉伯糖和 2,6-二氯嘌 呤缩合, 提高了反应的立体选择性 $(\beta / \alpha=21 / 1)$, 经氨解 和脱保护基之后可得氯法拉滨. 该方法虽然提高了 $\beta$ 和 $\alpha$-异构体的比例, 使分离操作更为容易, 但仍需经过 6 步, 总收率也仅为 $14 \%$. 上述两条路线主要缺点是: (1) 所用原料溴代糖不是商品化的原料, 一般需 $4 \sim 6$ 步才 能合成, 且性质不稳定 ${ }^{[9]} ;(2)$ 合成路线步骤长, 总收率 低. 2012 年, Henschke 课题组 ${ }^{[10]}$ 使用较为廉价的 1-O-乙 酰基-2,3,5-三- $O$-苯甲酰基- $\alpha$ - $D$-㫙喃核糖代替上述方法 中的溴代糖, 和 2 -氯腺嘌呤缩合, 然后选择性地脱除 $2^{\prime}$ 位的苯甲酰基, 最后对 $2^{\prime}$ 位羟基进行三氟甲磺酰化和氟 化取代反应可得氯法拉滨(图 2, 路线 c). 这个路线包括 7 步反应，总收率为 $12 \%$, 对比其它方法来讲没有优势. 因此, 需要发展一种步骤短、总收率高、无需分离 $\beta$ 和 $\alpha$-型产品和原料廉价易得的方法来合成氯法拉宾.

(a) 6 steps, $6 \%$<smiles>CC(=O)OCC1OC(Br)C(OC(=O)OCc2ccccc2)C1Br</smiles><smiles>Clc1nc(Cl)c2nc[nH]c2n1</smiles>

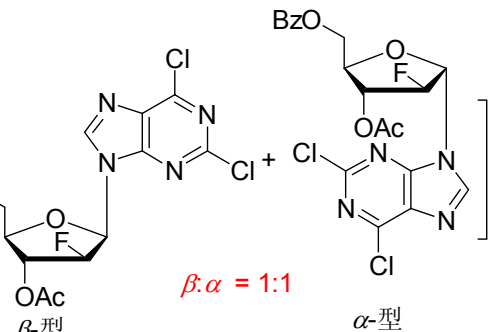

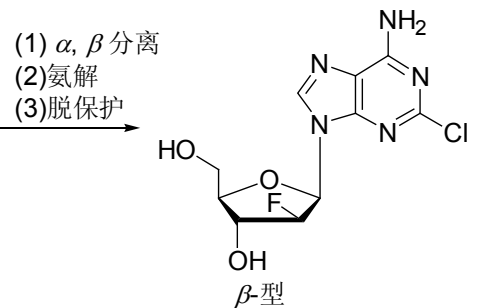

(b) 6 steps, $14 \%$

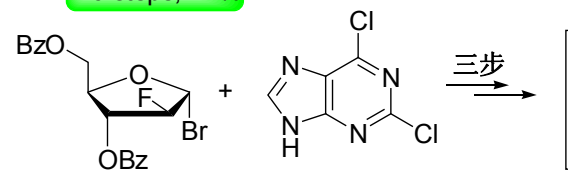

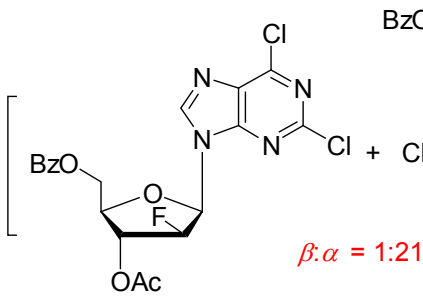
$\beta$-型

$\mathrm{BzO}$

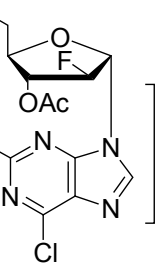

(1) $\alpha, \beta$ 分离

(2)重结晶 (3)脱保护

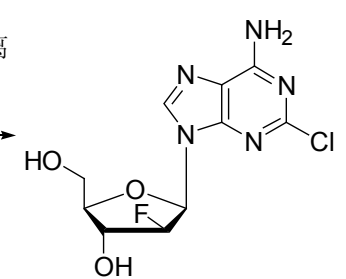
$\alpha$-型 $\beta$-型

(c) 7 steps, $12 \%$

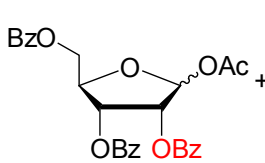<smiles>Nc1nc(Cl)nc2[nH]cnc12</smiles>

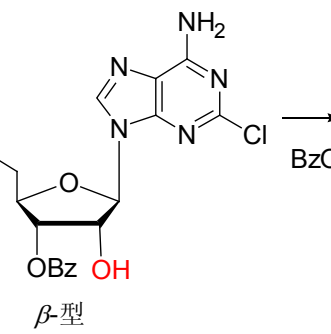

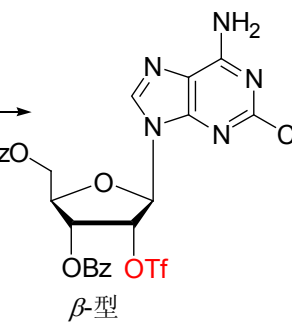

(1) $\mathrm{KF} / 18-$ Crown-6 (2) 脱保护<smiles>Nc1nc(Cl)nc2c1ncn2C1CC2CCC(O2)C1O</smiles>

图 2 氯法拉滨的合成路线

Figure 2 Synthetic routes to clofarabine 
上述方法(图 2, 路线 $\mathrm{a} \sim \mathrm{c}$ )都用到了糖基和嘌呤碱 基的缩合. 由于氟原子半径很小, 接近于氢原子, 所以 $2^{\prime}$ 位的氟原子在缩合过程中不能有效控制产物的立体选 择性, 从而得到 $\alpha / \beta$-构型的混合物, 造成分离困难和较 低的收率. 我们设想, 如果起始原料全部是 $\beta$-构型, 在 反应过程中不涉及 $\alpha / \beta$-构型的转化, 那么得到的产物就 必然全是单一的 $\beta$-构型产物. 我们在以往核苷类化合物 的修饰和产业化研究的基础上 ${ }^{[11,12]}$, 发展了以单一 $\beta$-构 型的 $\beta$-氯腺苷为原料, 用乙酰基保护糖环上的三个羟 基, 通过选择性脱除 $2^{\prime}$ 位的乙酰基, 裸露出 $2^{\prime}$ 位的羟基, 然后用氟代试剂乙胺基三氟化硫(DAST)氟化取代, 同 时氟原子位置翻转, 最终以 4 步和 $49 \%$ 的总收率得到氯 法拉滨(图 3). 该方法步骤少, 收率高, 构型单一, 并且 可以进行克级规模的实验室合成, 具有较好的应用价 值.

\section{1 结果与讨论}

\subsection{2 '位乙酰基的选择性脱除}

本方法的关键步骤是 $2^{\prime}$ 位乙酰基的选择性脱除. 在 三乙酰基保护的核苷中，2'位乙酰基的选择性脱除是糖 基修饰的重要策略. 2 '位羟基裸露出来以后, 可以对其 进行脱羟基 ${ }^{[13]}$ 和磷酸化 ${ }^{[14]}$ 等修饰, 以得到活性更好的 核苷类化合物. 1,3-二氯-1,1,3,3-四异丙基-1,3-二硅烷 $(\mathrm{TPDSCl})^{[13]}$ 可以选择性地保护糖环上的 3',5羟基, 从而 将 2'羟基裸露出来. 但是, TPDSCl 价格昂贵, 导致该方 法难以推广. Ishido 课题组 ${ }^{[15]}$ 曾报道过用乙酸和水合肼
在吡啶溶液中, 或用醋酸差忽在吡定溶液中脱除 2 '位酰 基的方法，但是底物适应性差，收率也不高. 其它选择 性脱酰基的试剂，如三氟乙醇锂 ${ }^{[16]}$ 等也有报道，但更适 应于脱除 $5^{\prime}$ 位的酰基.

在上述选择性脱除酰基的方法中, Ishido 课题组发 展的方法所用试剂价格便宜. 受此启发, 我们先将原料 2-氯腺苷糖基上的羟基用乙酰基保护, 然后参考 Ishido 课题组的方法选择性脱除 $2^{\prime}$ 位的乙酰基. 首先考查不同 的脱酰基试剂对反应的影响时. 在 2.0 equiv.乙酸和 2.0 equiv.水合肼作用下，室温反应 $4 \mathrm{~h}$, 基本没有产物生成 (表 1, Entry 1). 当将水合肼用量增加到 4.0 equiv., 室温 反应 $4 \mathrm{~h}, 2$ '位酰基脱除产物 4 的收率达到 $65 \%$, 伴随有 部分原料剩余(表 1, Entry 2). 当反应时间延长至 $24 \mathrm{~h}$, 产物 4 的收率反而有所下降, 并伴随有脱掉两个酰基的 产物(表 1 , Entry 3). 随后, 降低反应温度至 $0{ }^{\circ} \mathrm{C}$, 反应 $24 \mathrm{~h}$ 后，产物 4 收率可达 $72 \%$, 并且原料基本反应完全 (表 1, Entry 4). 接下来, 对水合肼的用量进行了筛选, 减少水合肼的用量, 产物 $\mathbf{4}$ 的收率会大幅下降. 增加水 合肼的用量, 会导致副产物的生成, 产物 4 的收率有所 降低(表 1, Entries 5 6). 因此, 选择 2.0 equiv. 的水合肼 最为合适(表 1, Entry 4). 对于文献中报道过的乙酸羟氨 或三氟乙醇锂, 无论在室温还是 $0{ }^{\circ} \mathrm{C}$ 下反应, 产物 $\mathbf{4}$ 的 收率都比较低(表 1, Entries 7 9).

对于选择性脱除 2 '位乙酰基，在最佳反应条件下 (表 1, Entry 4), 我们将反应规模从 $0.5 \mathrm{mmol}$ 扩大到 10 $\mathrm{mmol}$ 和 $100 \mathrm{mmol}$. 反应结果显示: 反应收率并没有下
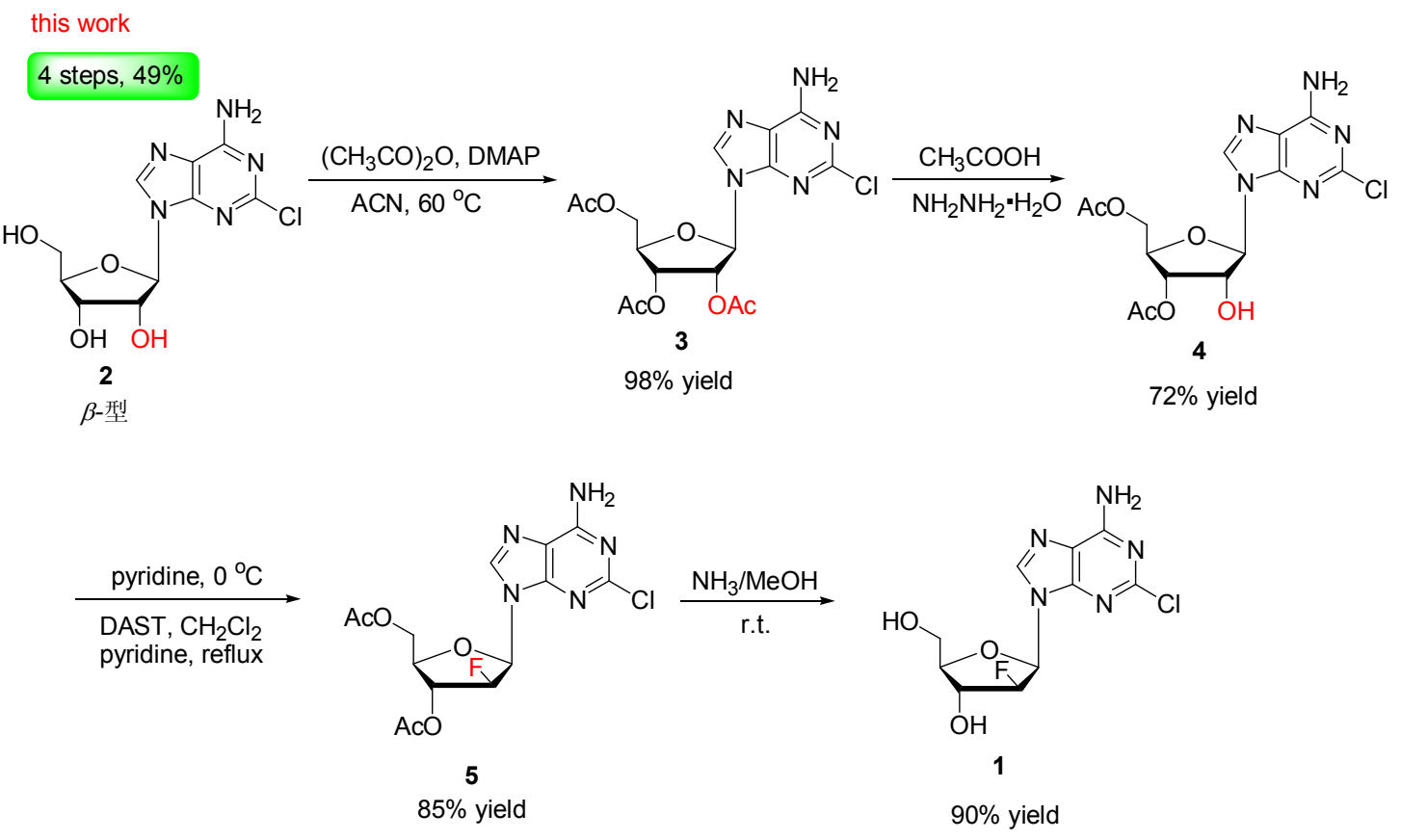

图 3 以 2-氯腺苷为原料制备氯法拉滨的合成路线

Figure 3 Synthesis route for clofarabine from 2-chloroadenosine 
表 12 '位脱除乙酰基的条件优化 ${ }^{a}$

Table 1 The optimization for the deacetylation reaction condition of 2'-position ${ }^{a}$

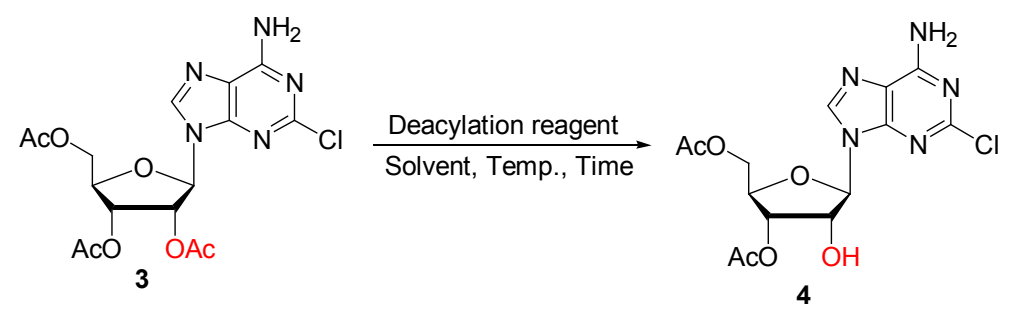

\begin{tabular}{cllccc}
\hline Entry & \multicolumn{1}{c}{ Deacylation reagent } & \multicolumn{1}{c}{ Solvent } & Temp. $/{ }^{\circ} \mathrm{C}$ & Time $/ \mathrm{h}$ & Yield $/ \%$ \\
\hline 1 & $\mathrm{CH}_{3} \mathrm{COOH} /$ hydrazine hydrate $(1 / 1)$ & Pyridine & r.t. & 4 & Trace \\
2 & $\mathrm{CH}_{3} \mathrm{COOH} /$ hydrazine hydrate $(1 / 2)$ & Pyridine & r.t. & 4 & 65 \\
3 & $\mathrm{CH}_{3} \mathrm{COOH} /$ hydrazine hydrate $(1 / 2)$ & Pyridine & r.t. & 24 & 32 \\
$\mathbf{4}$ & $\mathrm{CH}_{3} \mathrm{COOH} /$ hydrazine hydrate $(1 / 2)$ & Pyridine & 0 & $\mathbf{2 4}$ & $\mathbf{7 2}$ \\
5 & $\mathrm{CH}_{3} \mathrm{COOH} /$ hydrazine hydrate $(1 / 1)$ & Pyridine & 0 & 24 & 16 \\
6 & $\mathrm{CH}_{3} \mathrm{COOH} /$ hydrazine hydrate $(1 / 3)$ & Pyridine & rt & 24 & 34 \\
$7^{c}$ & Hydroxylammonium acetate $(2.0 \mathrm{mmol})$ & Pyridine & 0 & 24 & 32 \\
$8^{c}$ & Hydroxylammonium acetate $(2.0 \mathrm{mmol})$ & Pyridine & r.t. & 24 & 15 \\
$9^{c}$ & Lithium trifluoroethoxide $(2.0 \mathrm{mmol})$ & Trifluoroethanol & & 23 \\
\hline
\end{tabular}

${ }^{a}$ Unless otherwise noted, the reaction conditions was: $3(0.50 \mathrm{mmol})$, solvent $(10.0 \mathrm{~mL})$, deacetylation reagent $(2.0$ equiv. $) ;{ }^{b}$ isolated yield; ${ }^{c}$ deacetylation reagent (4.0 equiv).

降(图 4). 值得一提的是：当反应量增大以后，产物 $\mathbf{4}$ 可 以通过结晶的方法来纯化. 即不需要通过柱层析, 即可 纯化产品 $\mathbf{4}$, 这更加符合工业化的要求. 与此同时, 对 脱除酰基后的废液重新乙酰化, 可以实现原料 $\mathbf{3}$ 的回收 利用.

由于选择性脱除 $2^{\prime}$ 位的乙酰基是基于糖环上三个乙 酰基性质的微小差别 ${ }^{[16]}$, 然而, 关于碱基取代基对选择 性脱除酰基反应的影响, 至今没有报道. 在得到 2 '位脱 除乙酰基的最佳反应条件之后，我们首次考查了碱基 2 位上的取代基对反应的影响(图 5). 在最佳反应条件下, 所考查的底物都能以中等的收率得到相应的选择性脱 除酰基的产物.

\subsection{2 '位羟基的氟代}

得到 2 '位脱酰基的产物 4 之后, 我们以 DAST 为氟 代试剂对 $2^{\prime}$ 位着基进行氟代反应. 如表 2 所示, 当使用 三乙胺时, 室温反应 $2 \mathrm{~h}$, 基本没有氟代产物 5 生成(表
2, Entry 1). 升高反应温度, 能够以 $29 \%$ 的收率得到氟代 产物 $\mathbf{5}$ (表 2, Entry 2). 当用吡啶代替三乙胺, 氟代产物 $\mathbf{5}$ 的收率可以提高到 50\% (表 2, Entry 3). 接下来对 DAST 的用量和反应时间进行考查(表 1, Entries 4 8), 结果显 示: 使用 1.5 equiv. DAST，回流条件下反应 $6 \mathrm{~h}$, 能够以 85\%的收率得到氟代产物 5 (表 1, Entry 6).

接下来, 我们将反应规模扩大至 $100 \mathrm{mmol}$ (图 6), 反应也能很好的进行, 延长反应时间至 $10 \mathrm{~h}$, 能够以 80\%的收率得到氟代产物 5 . 最终，除去其它的保护基 之后, 我们以 4 步和 $49 \%$ 的总收率得到了氯法拉滨. 同 时，当反应放大到克级之后，该方法仍然可行(图 6).

\section{2 结论}

发展了一种有效合成氯法拉滨的新方法. 该方法以 廉价且商品化的 2-氯腺苷为原料, 通过乙酰基将糖环上 的羟基保护之后，使用乙酸和水合肼实现选择性脱除 $2^{\prime}$
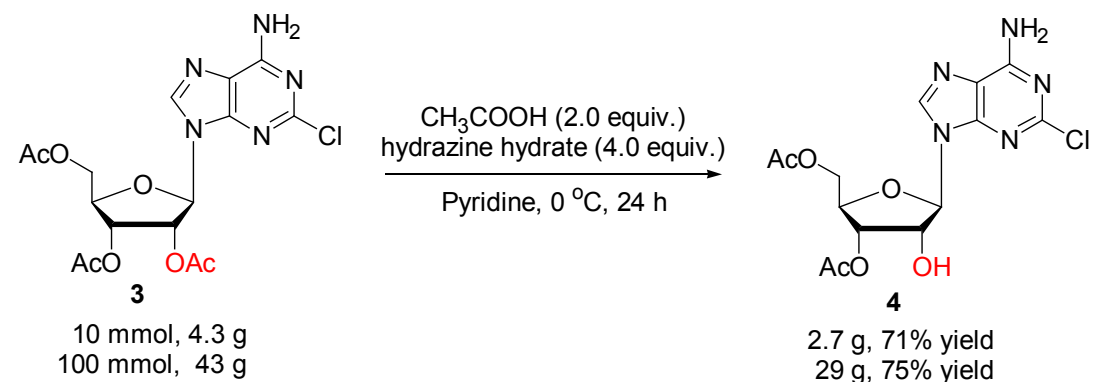

图 4 克级规模下的脱除酰基反应

Figure 4 The gram-scale experiments of deacetylation 


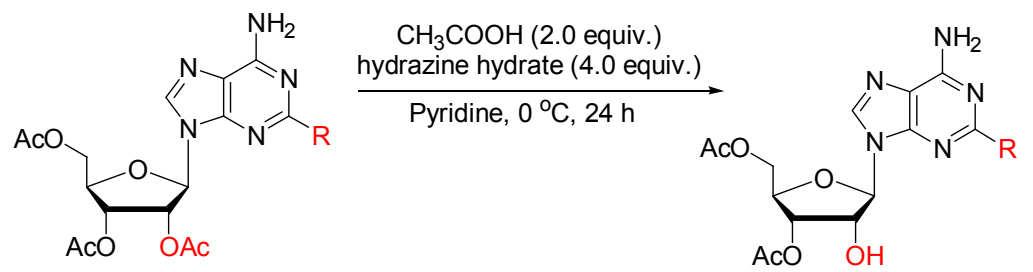

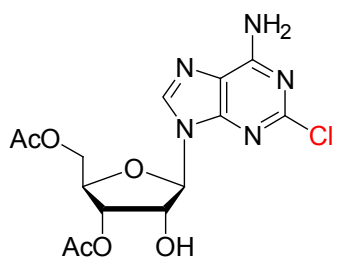

$4,72 \%$ yield

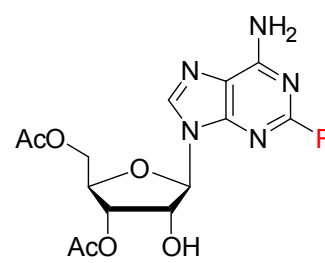

6, $60 \%$ yield

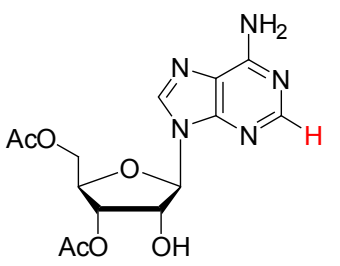

$7,54 \%$ yield

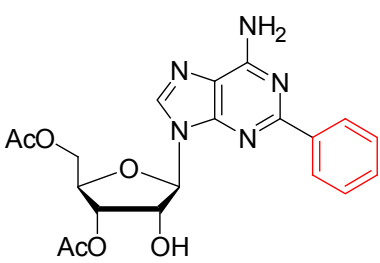

8, $73 \%$ yield

图 5 嘌呤 2 位取代基对选择性脱除乙酰基的影响

Figure 5 The effects of 2-substitution of purines for the deacetylation reaction

表 2 氟代步骤反应条件的优化 ${ }^{a}$

Table 2 The optimization of reaction condition for fluorination step<smiles>CC(=O)OCC1OC(n2cnc3c(N)nc(C)nc32)C(O)C1OC(C)=O</smiles>
$\underset{\text { Temp., Time }}{\stackrel{\text { base, DAST }}{\longrightarrow}}$

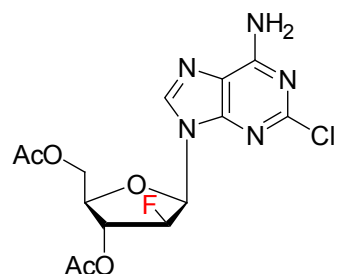

5

\begin{tabular}{clcccc}
\hline Entry & Base (equiv.) & DAST/equiv. & Temp. $/{ }^{\circ} \mathrm{C}$ & Time $/ \mathrm{h}$ & Yield $/ \%$ \\
\hline 1 & TEA (6.0) & 1.5 & r.t. & 2 & Trace \\
2 & TEA (6.0) & 1.5 & Reflux & 2 & 29 \\
3 & Pyridine (6.0) & 1.2 & Reflux & 2 & 50 \\
4 & Pyridine (6.0) & 1.2 & Reflux & 4 & 64 \\
5 & Pyridine (6.0) & 1.5 & Reflux & 4 & 75 \\
$\mathbf{6}$ & Pyridine (6.0) & $\mathbf{1 . 5}$ & Reflux & $\mathbf{6}$ & $\mathbf{8 5}$ \\
7 & Pyridine (6.0) & 1.5 & Reflux & 8 & 76 \\
8 & Pyridine (6.0) & 1.8 & 6 & 60 \\
\hline
\end{tabular}

${ }^{a}$ Unless otherwise noted, the reaction conditions was: $4(2.46 \mathrm{mmol}), \mathrm{CH}_{2} \mathrm{Cl}_{2}(50.0 \mathrm{~mL}) ;{ }^{b}$ isolated yield.

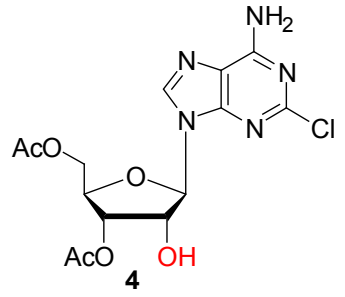

$2.46 \mathrm{mmol}, 1 \mathrm{~g}, 6 \mathrm{~h}$ $100 \mathrm{mmol}, 41 \mathrm{~g}, 10 \mathrm{~h}$

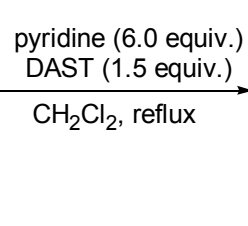

$\underset{\mathrm{CH}_{2} \mathrm{Cl}_{2} \text {, reflux }}{\mathrm{DAST} \text {. }}$

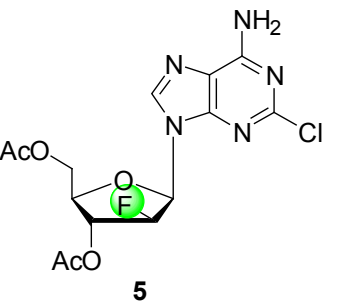

$0.81 \mathrm{~g}, 85 \%$ yield

$31 \mathrm{~g}, 80 \%$ yield

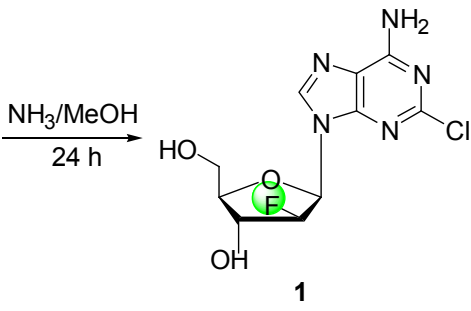

$0.67 \mathrm{~g}, 90 \%$ yield

$28 \mathrm{~g}, 92 \%$ yield

图 6 不同反应规模的影响

Figure 6 The scale effects for the yield

位的乙酰基. 再以氟化试剂 DAST 对 $2^{\prime}$ 位的羟基进行氟 化取代，通过脱除剩下的乙酰基之后，即可得到氯法拉
宾. 该路线仅需 4 步，且总收率可以达到 $49 \%$. 相比与 传统方法，缩短了合成步骤，提高了反应的总收率，且 
构型单一，原料廉价易得. 同时，该反应规模可以放大 到到克级, 收率可以很好的保持, 而且中间体和产物可 以通过结晶的方法进行纯化, 有着良好的应用前景.

\section{3 实验部分}

\section{1 仪器与试剂}

高分辨质谱用 Waters Q-TofMS/MS 测定. 核磁共振 仪: Bruker AM 400, 以 TMS 为内标, 氞代氯仿为溶剂. 熔点仪为: X-4 型数字熔点仪. 所用的药品和试剂均为 市售分析纯或化学纯, 除特别说明外, 未经进一步处理.

\section{$3.22^{\prime}, 3^{\prime}, 5^{\prime}$-三-O-乙酰基-2-氯腺苷(3)的合成}

2-氯腺苷 $(2,2.00 \mathrm{~g}, 6.62 \mathrm{mmol}), 4$-二胺甲基吡啶 (DMAP, $0.04 \mathrm{~g}, 0.33 \mathrm{mmol}, 5 \mathrm{~mol} \%$ ) 和三乙胺(TEA, 3.70 $\mathrm{mL}, 26.60 \mathrm{mmol})$ 悬浮于乙腈 $(50.0 \mathrm{~mL})$ 中, 摚拌 $10 \mathrm{~min}$, 滴加乙酸䣶 $(2.2 \mathrm{~mL}, 23.27 \mathrm{mmol})$. 滴加完毕后, 升温至 $50{ }^{\circ} \mathrm{C}$, 并在该温度下反应 $1 \mathrm{~h}$. TLC 检测反应, 显示原 料消失. 新生成的斑点单一 $[V$ (氯仿 $): V$ (甲醇 $)=9: 1$, $\left.R_{\mathrm{f}}=0.52\right]$. 加入甲醇 $(2.0 \mathrm{~mL})$ 终止反应, 真空浓缩, 除去 溶剂. 加入乙醇 $(5.0 \mathrm{~mL})$, 继续摚拌, 直到有晶体析出. 过滤、干燥后, 即得产物 3. 白色固体, $2.78 \mathrm{~g}$, 收率 $98 \%$. m.p. $150 \sim 152{ }^{\circ} \mathrm{C} \quad\left(\right.$ lit. $\left.{ }^{[17]} 151 \sim 152{ }^{\circ} \mathrm{C}\right)$; ${ }^{1} \mathrm{H}$ NMR $\left(\mathrm{CDCl}_{3}, 400 \mathrm{MHz}\right) \delta: 7.97$ (s, 1H), 6.96 (brs, 2H), $6.16(\mathrm{~d}$, $J=5.6 \mathrm{~Hz}, 1 \mathrm{H}), 5.78(\mathrm{t}, J=5.6 \mathrm{~Hz}, 1 \mathrm{H}), 5.59 \sim 5.57(\mathrm{~m}$, 1H), $4.42 \sim 4.37$ (m, 3H), 2.13 (s, 3H), 2.10 (s, 3H), 2.06 (s, 3H); ${ }^{13} \mathrm{C}$ NMR $\left(\mathrm{CDCl}_{3}, 100 \mathrm{MHz}\right) \delta: 170.5,169.7$, 169.6, 156.4, 154.4, 150.6, 138.8, 118.5, 85.8, 80.4, 73.2, 70.6, 63.1, 20.8, 20.6, 20.5; HRMS calcd for $\mathrm{C}_{16} \mathrm{H}_{19} \mathrm{Cl}-$ $\mathrm{N}_{5} \mathrm{O}_{7}\left[\mathrm{M}+\mathrm{H}^{+}\right]$428.0968, found 428.0966 .

\section{$3.33^{\prime}, 5^{\prime}$-二-O-乙酰基-2-氯腺苷(4)的合成}

乙酸 $(2.7 \mathrm{~mL}, 46.7 \mathrm{mmol}), 85 \%$ 水合肼 $(5.3 \mathrm{~mL}, 93.4$ $\mathrm{mmol})$ 和吡啶 $(85.0 \mathrm{~mL})$ 加入到反应瓶中, 温度降至 0 ${ }^{\circ} \mathrm{C}$, 慢慢加入 $2^{\prime}, 3^{\prime}, 5^{\prime}$-三- $O$-乙酰基-2-氯腺苷 $(3,10 \mathrm{~g}$, $23.36 \mathrm{mmol}$ ), 继续反应 $24 \mathrm{~h}$. 反应完成后, 加入丙酮 $(20.0 \mathrm{~mL})$ 终止反应. 真空浓缩后, 加入甲醇 $(65.0 \mathrm{~mL})$, 并继续在 $0{ }^{\circ} \mathrm{C}$ 搅拌, 约 $2 \mathrm{~h}$ 后有白色固体析出. 过滤、 干燥后即得产品 4. 白色固体, $6.80 \mathrm{~g}, \mathrm{HPLC}$ 纯度 $95 \%$, 收率 72\%. m.p. $184 \sim 186{ }^{\circ} \mathrm{C} ;{ }^{1} \mathrm{H}$ NMR (DMSO- $d_{6}, 400$ MHz) $\delta: 8.37$ (s, 1H), 7.86 (brs, 2H), 5.84 (d, $J=6.4 \mathrm{~Hz}$, $1 \mathrm{H}), 5.26 \sim 5.24(\mathrm{~m}, 1 \mathrm{H}), 4.90(\mathrm{t}, J=5.6 \mathrm{~Hz}, 1 \mathrm{H}), 4.33 \sim$ $4.21(\mathrm{~m}, 3 \mathrm{H}), 2.11(\mathrm{~s}, 3 \mathrm{H}), 2.03(\mathrm{~s}, 3 \mathrm{H}) ;{ }^{13} \mathrm{C} \mathrm{NMR}$ (DMSO- $\left.d_{6}, 100 \mathrm{MHz}\right) \delta: 170.6,170.1,157.3,153.7,150.9$, $140.6,118.7,87.7,80.0,72.8,71.5,63.7,21.2,21.0$; HRMS calcd for $\mathrm{C}_{14} \mathrm{H}_{17} \mathrm{ClN}_{5} \mathrm{O}_{6}\left[\mathrm{M}+\mathrm{H}^{+}\right] 386.0862$, found 386.0865 .

\section{$3.43^{\prime}, 5^{\prime}$-二-O-乙酰基-2'- $\alpha$-氟-2-氯腺苷(5)的合成}

3',5'-二- $O$-乙酰基- 2 -氯腺苷 $(4,1.0 \mathrm{~g}, 2.46 \mathrm{mmol})$, 吡 啶 $(1.2 \mathrm{~mL}, 14.40 \mathrm{mmol})$ 加入到 $\mathrm{CH}_{2} \mathrm{Cl}_{2}(20.0 \mathrm{~mL})$ 中, 冷 至 $0{ }^{\circ} \mathrm{C}$ 以下, 缓慢滴加 DAST $(0.5 \mathrm{~mL}, 3.90 \mathrm{mmol})$, 滴 加结束后, 升至室温, 加热至回流并反应 $6 \mathrm{~h}, \mathrm{TLC}$ 检测 反应. 反应完成后, 反应液冷至室温, 用饱和 $\mathrm{NaHCO}_{3}$ 水溶液洗涤, 收集有机相. 使用活性炭脱色, 并真空除 去溶剂. 最后, 加入甲醇 $(5.0 \mathrm{~mL})$ 结晶、过滤、干燥后, 即 得产品 5. 白色固体, $0.81 \mathrm{~g}$, 收率 $85 \%$. m.p. 190 192 ${ }^{\circ} \mathrm{C} ;{ }^{1} \mathrm{H}$ NMR $\left(\mathrm{CDCl}_{3}, 400 \mathrm{MHz}\right) \delta: 8.04$ (s, $\left.1 \mathrm{H}\right), 6.88$ (brs, $2 \mathrm{H}), 6.47\left(\mathrm{~d}, J_{\mathrm{C}-\mathrm{F}}=22.4 \mathrm{~Hz}, 1 \mathrm{H}\right), 5.35(\mathrm{~d}, J=16 \mathrm{~Hz}, 1 \mathrm{H})$, $5.19\left(\mathrm{~d}, J_{\mathrm{C}-\mathrm{F}}=50 \mathrm{~Hz}, 1 \mathrm{H}\right), 4.48 \sim 4.39(\mathrm{~m}, 2 \mathrm{H}), 4.27(\mathrm{~s}$, $1 \mathrm{H}), 2.17(\mathrm{~s}, 3 \mathrm{H}), 2.11$ (s, 3H); ${ }^{13} \mathrm{C}$ NMR (DMSO- $d_{6}, 100$ MHz) $\delta: 170.6,170.1,157.3,153.9,140.7,94.0,92.1$, 82.3, 82.1, 78.6, 75.8, 75.7, 63.5, 21.1, 21.0; HRMS calcd for $\mathrm{C}_{14} \mathrm{H}_{15} \mathrm{ClFN}_{5} \mathrm{NaO}_{5}\left[\mathrm{M}+\mathrm{Na}^{+}\right]$410.0638, found 410.0640 .

\section{5 氯法拉滨(1)的合成}

3',5'-二- $O$-乙酰基-2'- $\alpha$-氟- 2 -氯腺苷 $(5,1.0 \mathrm{~g}, 2.58$ $\mathrm{mmol}$ )加入到 $\mathrm{NH}_{3} / \mathrm{MeOH}$ 饱和溶液 $(100.0 \mathrm{~mL}$ )中, 室温 摚拌 $12 \mathrm{~h}$, 真空浓缩, 乙酸乙酯重结晶, 白色固体, 0.71 g, 收率 90\%. m.p. $236 \sim 238{ }^{\circ} \mathrm{C}$ (lit. ${ }^{[7]}$ m.p. $237{ }^{\circ} \mathrm{C}$ ); ${ }^{1} \mathrm{H}$ NMR (DMSO- $\left.d_{6}, 400 \mathrm{MHz}\right) \delta: 8.26(\mathrm{~d}, J=1.6 \mathrm{~Hz}, 1 \mathrm{H})$, 7.98 (brs, $2 \mathrm{H}), 6.34 \sim 6.29(\mathrm{~m}, 1 \mathrm{H}), 5.95(\mathrm{~d}, J=4.8 \mathrm{~Hz}$, $1 \mathrm{H}), 5.29(\mathrm{~d}, J=8 \mathrm{~Hz}, 1 \mathrm{H}), 5.08(\mathrm{~d}, J=5.6 \mathrm{~Hz}, 1 \mathrm{H})$, $4.46 \sim 4.38(\mathrm{~m}, 1 \mathrm{H}), 3.84(\mathrm{~d}, J=4.4 \mathrm{~Hz}, 1 \mathrm{H}), 3.70 \sim 3.39$ (m, $2 \mathrm{H}$ ); ${ }^{13} \mathrm{C}$ NMR (DMSO- $\left.d_{6}, 100 \mathrm{MHz}\right) \delta: 157.2,153.7$, 150.6, 140.2, 117.8, 96.7, 94.8, 84.0, 83.9, 82.0, 81.8, 73.1, $72.9,60.8 ;{ }^{19} \mathrm{~F}$ NMR (DMSO- $d_{6}, 376 \mathrm{MHz}$ ), $\delta:-198.1$; HRMS calcd for $\mathrm{C}_{10} \mathrm{H}_{12} \mathrm{ClFN}_{5} \mathrm{O}_{3}\left[\mathrm{M}+\mathrm{H}^{+}\right]$304.0607, found 304.0609.

\section{6 在化合物 4 的条件下合成化合物 $6 \sim 8$}

3',5'-二- $O$-乙酰基-2-氟腺苷 $(6)$ : 无色油状物, 产率 $60 \%$. ${ }^{1} \mathrm{H}$ NMR $\left(\mathrm{CDCl}_{3}, 400 \mathrm{MHz}\right) \delta: 8.26(\mathrm{~s}, 1 \mathrm{H}), 8.01$ (brs, $2 \mathrm{H}), 6.17(\mathrm{~d}, J=5.6 \mathrm{~Hz}, 1 \mathrm{H}), 5.82(\mathrm{t}, J=4.2 \mathrm{~Hz}, 1 \mathrm{H})$, $5.57(\mathrm{~d}, J=4.8 \mathrm{~Hz}, 1 \mathrm{H}), 4.44 \sim 4.36(\mathrm{~m}, 3 \mathrm{H}), 3.42$ (brs, $1 \mathrm{H}), 2.12(\mathrm{~s}, 3 \mathrm{H}), 2.10(\mathrm{~s}, 3 \mathrm{H}) ;{ }^{13} \mathrm{C}$ NMR $\left(\mathrm{CDCl}_{3}, 100\right.$ MHz) $\delta: 169.6,169.4,160.4,158.3,157.4,157.2,151.2$, 151.0, 138.7, 117.8, 86.1, 80.5, 73.1, 70.6, 63.0, 20.7, 20.5; HRMS calcd for $\mathrm{C}_{14} \mathrm{H}_{17} \mathrm{FN}_{5} \mathrm{O}_{6}\left[\mathrm{M}+\mathrm{H}^{+}\right] 370.1157$, found 370.1159 .

3',5'-二-O-乙酰基腺苷(7): 白色固体，产率 $54 \%$. m.p. $172 \sim 174{ }^{\circ} \mathrm{C}$ (lit. ${ }^{[18]}$ m.p. $172 \sim 173{ }^{\circ} \mathrm{C}$ ); ${ }^{1} \mathrm{H}$ NMR $\left(\mathrm{CDCl}_{3}, 400 \mathrm{MHz}\right) \delta: 8.33(\mathrm{~s}, 1 \mathrm{H}), 7.95(\mathrm{~s}, 1 \mathrm{H}), 6.18(\mathrm{~d}$, 
$J=5.2 \mathrm{~Hz}, 1 \mathrm{H}), 6.14$ (brs, 2H), 5.92 (t, $J=5.2 \mathrm{~Hz}, 1 \mathrm{H}$ ), $5.66(\mathrm{t}, J=4.4 \mathrm{~Hz}, 1 \mathrm{H}), 4.45 \sim 4.42(\mathrm{~m}, 2 \mathrm{H}), 4.38 \sim 4.33$ (m, 1H), 2.12 (s, 3H), 2.09 (s, 3H); ${ }^{13} \mathrm{C} \mathrm{NMR}\left(\mathrm{CDCl}_{3}, 100\right.$ MHz) $\delta: 169.6,169.4,155.6,153.0,149.7,138.9,120.1$, 86.3, 80.3, 73.2, 70.7, 63.1, 20.7, 20.5; HRMS calcd for $\mathrm{C}_{14} \mathrm{H}_{18} \mathrm{~N}_{5} \mathrm{O}_{6}\left[\mathrm{M}+\mathrm{H}^{+}\right]$352.1252, found 352.1250.

3',5'-二- $O$-乙酰基-2-苯基腺苷 (8): 无色油状物, 产 率 73\%. ${ }^{1} \mathrm{H}$ NMR $\left(\mathrm{CDCl}_{3}, 400 \mathrm{MHz}\right) \delta: 7.94(\mathrm{~s}, 1 \mathrm{H})$, $7.33 \sim 7.27$ (m, 5H), 6.66 (brs, 2H), 6.17 (d, $J=5.2 \mathrm{~Hz}$, $1 \mathrm{H}), 5.78$ (t, $J=5.2 \mathrm{~Hz}, 1 \mathrm{H}), 5.60(\mathrm{~d}, J=4.4 \mathrm{~Hz}, 1 \mathrm{H})$, $4.43 \sim 4.38(\mathrm{~m}, 3 \mathrm{H}), 3.22$ (brs, $1 \mathrm{H}), 2.15(\mathrm{~s}, 3 \mathrm{H}), 2.13$ (s, $3 \mathrm{H}) ;{ }^{13} \mathrm{C}$ NMR $\left(\mathrm{CDCl}_{3}, 100 \mathrm{MHz}\right) \delta: 169.5,169.3,156.2$, 150.3, 138.5, 129.2, 128.7, 127.9, 118.3, 85.5, 80.1, 72.9, 70.3, 62.8, 20.6, 20.3; HRMS calcd for $\mathrm{C}_{20} \mathrm{H}_{22} \mathrm{~N}_{5} \mathrm{O}_{6}[\mathrm{M}+$ $\left.\mathrm{H}^{+}\right]$428.1565, found 428.1568 .

辅助材料(Supporting Information) 合成步骤、产物及 中间体的核磁共振图. 这些材料可以免费从本刊网站 (http://sioc-journal.cn/)上下载.

\section{References}

[1] (a) Lee, S.; Uttamapinant, C.; Verdine, G. L. Org. Lett. 2007, 9, 5007.

(b) Chong, Y.; Choo, H.; Choi, Y.; Mathew, J.; Schinazi, R. F.; Chu, C. K. J. Med. Chem. 2002, 45, 4888.

[2] Xu, X.-H.; Qiu, X.-L.; Zhang, X.-G.; Qing, F.-L. J. Org. Chem. 2006, 71, 2820.

[3] Yamauchi, T.; Nowak, B. J.; Keating, M. J.; Plunkett, W. Clin. Cancer Res. 2001, 7, 3580.

[4] Kantargian, H. M.; Gandhi, V. V.; O'Brien, S.; Giles, F.; Coertes, J.; Kozuch, P.; Du, M.; Plunkett, W.; Rios, M. B.; Freireich, E. J.; Estey, E. H.; Keating, M. J. Blood 2001, 98, 214b Abstract 4568.

[5] Montgomery, J. A.; Shortnacy-Fowler, A. T.; Clayton, S. D.; Riordan, J. M.; Secrist III, J. A. J. Med. Chem. 1992, 35, 397.

[6] Montgomery, J. A.; Fowler, A. T.; Secrist III, J. A. WO 2001060383, 2001 [Chem. Abstr. 2001, 135, 180927].

[7] Bauta, W. E.; Schulmeier, B. E.; Burke, B.; Puente, J. F.; Cantrell, Jr.; Lovett, W. R. D.; Goebel, J.; Anderson, B.; Ionescu, D.; Guo, R.-C. Org. Process Res. Dev. 2004, 8, 889.

[8] Anderson, B. G.; Bauta, W. E.; Cantrell, Jr. W. R.; Engles, T.; Lovett, D. P. Org. Process Res. Dev. 2008, 12, 1229.
[9] (a) Wright, J. A.; Taylor, N. F.; Fox, J. J. J. Org. Chem. 1969, 34, 2632.

(b) Watanable, K. A.; Chu, C. K.; Fox, J. J.; Reichman, U.; Watanabe, K. A.; Fox, J. J. Carbohyd. Res. 1975, 42, 233.

[10] Henschke, J. P.; Zhang, X.-H.; Mei, L.-J.; Chen, Y.-F. US 20120010397, 2012 [Chem. Abstr. 2012, 156, 99519].

[11] (a) Guo, H.-M.; Wu, Y.-Y.; Niu, H.-Y.; Wang, D.-C.; Qu, G.-R. J. Org. Chem. 2010, 75, 3863.

(b) Guo, H.-M.; Xia, C.; Niu, H.-Y.; Zhang, X.-T.; Kong, S.-N.; Wang, D.-C.; Qu, G.-R. Adv. Synth. Catal. 2011, 353, 53.

(c) Guo, H.-M.; Yuan, T.-F.; Niu, H.-Y.; Liu, J.-Y.; Mao, R.-Z.; Li, D.-Y.; Qu, G.-R. Chem. Eur. J. 2011, 17, 4095.

(d) Niu, H.-Y.; Yuan, T.-F.; Qu, G.-R.; Li, D.-Y.; Mao, R.-Z.; Jin, X.; Yang, X.-N.; Guo, H.-M. Chin. J. Org. Chem. 2011, 31, 1613.

(e) Niu, H.-Y.; Zhang, X.-T.; Zhao, G.-Y.; Li, N.; Kong, S.-N.; Xia, C.; Qu, G.-R.; Guo, H.-M. Chin. J. Org. Chem. 2011, 31, 695.

(f) Wang, D.-C.; Niu, H.-Y.; Guo, H.-M.; Wei, X.-J.; Ding, R.-F.; Qu, G.-R. Chin. J. Org. Chem. 2012, 32, 1072.

(g) Meng, G.; Niu, H.-Y.; Qu, G.-R.; Fossey, J. S.; Li, J.-P.; Guo, H.-M. Chem. Commun. 2012, 48, 9601.

(h) Qu, G.-R.; Liang, L.; Niu, H.-Y.; Rao, W.-H.; Guo, H.-M.; Fossey, J. S. Org. Lett. 2012, 14, 4494.

(i) Xin, P.-Y.; Niu, H.-Y.; Qu, G.-R.; Ding, R.-F.; Guo, H.-M. Chem. Commun. 2012, 48, 6717.

(j) Li, J.-P.; Huang, Y.; Xie, M.-S.; Qu, G.-R.; Niu, H.-Y.; Wang, H.-X.; Qin, B.-W.; Guo, H.-M. J. Org. Chem. 2013, 78, 12629.

(k) Wang, D.-C.; Niu, H.-Y.; Xie, M.-S. Qu, G.-R.; Wang, H.-X.; Guo, H.-M. Org. Lett. 2014, 16, 262.

(1) Xia, R.; Xie, M.-S.; Niu, H.-Y.; Qu, G.-R.; Guo, H.-M. Org. Lett. 2014, 16, 444.

(m) Wei, T.; Xie, M.-S.; Qu, G.-R.; Niu, H.-Y.; Guo, H.-M. Org. Lett. 2014, 16, 900.

(n) Xie, M.-S.; Chu, Z.-L.; Qu, G..-R.; Niu, H.-Y.; Guo, H.-M. $J$. Org. Chem. 2014, 79, 1093.

[12] Guo, H.-M.; Wu, S.; Niu, H.-Y.; Song, G.; Qu, G.-R. In Chemical Synthesis of Nucleoside Analogues 3, Ed.: Merino, P., John Wiley \& Sons, New York, 2013, pp. 103 162 .

[13] Robins, M. J.; Wilson, J.; Sawyer, L.; James, M. G. Can. J. Chem. 1983, 61, 1911.

[14] Wagner, D.; Verheyden, J. H.; Moffatt, J. G. J. Org. Chem. 1974, 39,24 .

[15] Ishido, Y.; Sakairi, N.; Okazaki, K.; Nakazaki, N. J. Chem. Soc., Perkin Trans. 1 1980, 563.

[16] Nowak, I.; Jones, C. T.; Robins, M. J. J. Org. Chem. 2006, 71, 3077.

[17] Robins, M. J.; Uznański, B. Can. J. Chem. 1981, 59, 2601.

[18] Ishido, Y.; Nakazaki, N.; Sakairi, N. J. Chem. Soc., Perkin Trans. 1 1979, 2088. 